\title{
Interplay between Myasthenia Gravis and Severe COVID-19 Infection: The Missing Links
}

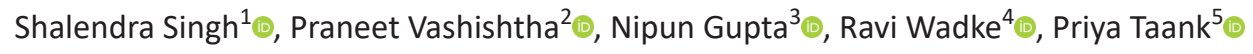

Keywords: COVID-19, Hydroxychloroquine, Intravenous immunoglobulin, Myasthenia gravis.

Indian Journal of Critical Care Medicine (2022): 10.5005/jp-journals-10071-23930

Sir,

Coronavirus disease (COVID-19) and myasthenia gravis (MG) disease spectrums range from asymptomatic presentation requiring no special treatment to severe form requiring ventilation with little knowledge about the association between two diseases. Higher morbidity and mortality rates are seen among the elderly and people with underlying comorbidities and immunologic deficiencies with disruption of immune and inflammatory processes being the shared mechanism behind it. Studies have shown considerable variability in how the MG patients respond to COVID-19.' However, it is unclear why some patients perform better than others, with likely prognostic factors being the timing of recognition and initiation of care. Here, we present a case of MG with simultaneous COVID-19 infection with an aim to illustrate the interplay between both diseases and discuss therapeutic approaches to maximize the outcome for this special set of patients.

A 63-year-old woman with a past medical history of hypertension, diabetes mellitus, and MG presented to us with symptoms of MG exacerbation consisting of fever, along with weakness and dyspnea at rest. She was maintained on tab metoprolol $25 \mathrm{mg}$ once in a day (OD), tab metformin $500 \mathrm{mg}$ $\mathrm{OD}$, tab prednisolone $25 \mathrm{mg} \mathrm{OD}$, and tab pyridostigmine $60 \mathrm{mg}$ thrice daily, and intravenous immunoglobulin G (IVIG) every 10 to 12 weeks along with worsening of weakness. With ongoing COVID-19 pandemic and patient's history of fever with respiratory symptoms, reverse transcriptase polymerase chain reaction (RT-PCR) analysis of her nasopharyngeal swab sample subsequently returned positive for COVID-19. In view of poor sensorium and desaturation, despite maximal oxygen support, the patient was intubated and placed on mechanical ventilation. Chest X-ray showed a patchy infiltrate in the left lower zone with bilateral minimal pleural effusion. Routine hematological and biochemical investigations were unremarkable except for anemia $(\mathrm{Hb}-8.6 \mathrm{gm} / \mathrm{dL})$ and leukocytosis (TLC-24000/ $\mathrm{LL})$. Cefepime, azithromycin, and remdesivir were used for COVID-19 infection and associated secondary infection; prednisolone, neostigmine, and intravenous immunoglobulins (IVIG) $(2 \mathrm{gm} / \mathrm{kg}$ over 5 days) were given for aggravation of MG; and subcutaneous enoxaparin in a dose of $60 \mathrm{mg}$ OD was started upon admission. Due to persistent hypoxemia despite full ventilator support, she was pronepositioned for 16 hours and supine-positioned for 8 hours, 5 days following admission. From day 01 onward, the patient responded to treatment in prone positioning showing a dramatic increase in oxygenation index and chest $\mathrm{X}$-ray. Monitoring of MG exacerbation ${ }^{1-4}$ Department of Anaesthesiology and Critical Care, Armed Forces
Medical College, Pune, Maharashtra, India
${ }^{5}$ Department of Ophthalmology, Command Hospital (SC), Pune,
Maharashtra, India

Corresponding Author: Shalendra Singh, Department of Anaesthesiology and Critical care, Armed Forces Medical College, Pune, Maharashtra, India, Phone: +9968619883, e-mail: drsinghafmc@ gmail.com

How to cite this article: Singh S, Vashishtha P, Gupta N, Wadke R, Taank P. Interplay between Myasthenia Gravis and Severe COVID-19 Infection: The Missing Links. Indian J Crit Care Med 2022;26(2):248-249.

Source of support: Nil

Conflict of interest: None

was limited by intubation and sedation. The patient was weaned off from mechanical ventilation over 2 days and was successfully extubated on the eighth day of admission. Post-extubation course of the patient was uneventful, and the patient was discharged on the 14th day of hospital admission.

MG with concurrent COVID-19 infection in a critical care setting can be challenging for wide plethora of reasons. MG patients are already at risk of infection due to immunosuppressive medication. When these patients contract COVID-19, there is potential respiratory failure in relation to respiratory muscle weakness and this can complicate the identification and subsequent management of the case and may result in a dire prognosis.

A panel of MG experts recently put forward guidelines for the management of MG during COVID-19. ${ }^{2}$ As per the recommendations of the panel, therapeutic management should be tailored as per individual patient's requirements. Additionally, the immunosuppressive medication should continue during mechanical ventilation, unless specifically discussed and advised against by an expert. Also, with increased dosage of corticosteroid being typically used as part of infection protocol and since the nature of COVID-19 infection in these cases is often severe, standard immunosuppressive agents that are long-acting, such as azathioprine and mycophenolate, can be tried. Immune-depleting agents should be avoided and stopped.

Currently, there is no evidence suggesting that IVIG or plasma exchange increases the risk of COVID-19 infections. IVIG uses pooled normal IgG that works through various mechanisms,

o The Author(s). 2022 Open Access This article is distributed under the terms of the Creative Commons Attribution 4.0 International License (https://creativecommons. org/licenses/by-nc/4.0/), which permits unrestricted use, distribution, and non-commercial reproduction in any medium, provided you give appropriate credit to the original author(s) and the source, provide a link to the Creative Commons license, and indicate if changes were made. The Creative Commons Public Domain Dedication waiver (http://creativecommons.org/publicdomain/zero/1.0/) applies to the data made available in this article, unless otherwise stated. 
such as blocking cytokine production, causing a reduction in endogenous/exogenous IgG, leading to a reduction in AChR antibodies, neutralization of autoantibodies, and inhibition of complement activation. The potential antiviral/ immunemodulating action of IVIG/hydroxychloroquine (HCQS) is under the scanner with a wide range of results. Additionally, there have been some reports of HCQS causing worsening of MG, and subsequently acute respiratory distress syndrome in COVID-19., This is even more common when administered in combination with macrolides, such as azithromycin, and may additionally necessitate dosage of IVIG. ${ }^{1}$ Administration of IVIG is also complicated by the potential for causing thrombosis. ${ }^{4}$ Extensive thrombosis has been documented in patients with COVID-19, and therefore, caution needs to be taken if IVIG is to be administered in patients with concomitant COVID-19.

The clinical management of a critically ill MG patient with severe COVID-19, especially those on ventilator support, poses various clinical dilemmas due to the potentially hazardous side effects of IVIG, HCQS, and acetylcholinesterase inhibitors, such as pyridostigmine. Ongoing registries of patients with COVID- 19 and MG should be useful in dealing with these singular challenges, particularly in cases necessitating mechanical ventilation as in this scenario. However, till the time a consensus has been reached, an individualized regimen seems best suited for patient care.

\section{ORCID}

Shalendra Singh (1) https://orcid.org/0000-0002-1112-3431

Praneet Vashishtha (1) https://orcid.org/0000-0002-9882-642X

Nipun Gupta (1) https://orcid.org/0000-0002-8467-9756

Ravi Wadke (1) https://orcid.org/0000-0002-2134-0990

Priya Taank ๑ https://orcid.org/0000-0002-4167-5376

\section{References}

1. Delly F, Syed MJ, Lisak RP, Zutshi D. Myasthenic crisis in COVID-19. J Neurol Sci 2020;414:116888. DOI: 10.1016/j.jns.2020.116888.

2. International MG/COVID-19 Working Group, Jacob S, Muppidi S, Guidon A, Guptill J, Hehir M, et al. Guidance for the management of myasthenia gravis (MG) and Lambert-Eaton myasthenic syndrome (LEMS) during the COVID-19 pandemic. J Neurol Sci 2020;412:116803. DOI: $10.1016 /$ j.jns.2020.116803.

3. Guidon AC, Amato AA. COVID-19 and neuromuscular disorders. Neurology 2020;94(22):959-969. DOI: 10.1212/WNL.000000 0000009566

4. Bonilla FA. Adverse effects of immunoglobulin $\mathrm{G}$ therapy: thromboembolism and haemolysis. Clin Exp Immunol 2014;178 (Suppl. 1):72-74. DOI: 10.1111/cei.12518. 